Touron, D. R., Hoyer, W. J., \& Cerella, J. (2001). Cognitive skill acquisition and transfer in younger and older adults. Psychology and Aging, 16(4): 555-563. (Dec 2001) Published by the American Psychological Association (ISSN: 1939-1498). This article may not exactly replicate the final version published in the APA journal. It is not the copy of record.

\title{
Cognitive Skill Acquisition and Transfer in Younger and Older Adults
}

Dayna R. Touron, William J. Hoyer, and John Cerella

\begin{abstract}
The acquisition of cognitive skills often depends on 1 of (or a combination of) 2 processes, the execution of an algorithm, and the retrieval of problem instances. This study examined the effects of age and repetition of problem instances on the production and verification of solutions to 2 serially presented sets of alphabet arithmetic problems. Analyses of the parameters derived from power-function fits for individuals revealed age differences favoring young adults in improvement span, learning rate, and asymptote. For both age groups, the beneficial effects of repetitions on 1st-set response times were attributable to algorithmic speedup and to the retrieval of instances, whereas improvements in the speed of 2nd-set response times were attributable primarily to item retrieval.
\end{abstract}


When individuals practice cognitive skills that require using a prescribed set of mental operations to obtain solutions, performance improves as a result of increased speed in carrying out the prescribed algorithm. Further, when individuals practice cognitive skills that involve repetitions of particular problems, improvements in performance can derive from the representation and retrieval of problem instances that have been previously presented. One aim of the present study was to examine the influences of these factors as possible sources of age differences in cognitive skill acquisition. Specifically, we describe the effects of familiarity with an algorithm and the effects of repetition of items on performance in an alphabet arithmetic task in which solutions to problems could be either computed or retrieved. Alphabet arithmetic was selected as the task domain for this experiment (a) because problems can be solved either by computation using a prescribed algorithm or by retrieval of prior instances and (b) because it is unlikely that younger and older participants had any experience with alphabet arithmetic tasks prior to our experimental testing.

Age-related differences in the effects of practice on cognitive performance are often attributed to deficits in the speed or efficiency of the computational or control processes involved in learning. For example, it has been suggested that age differences in the speed of completing elementary operations or in the loss of information derived from component steps contribute to overall deficits in learning (e.g., Charness \& Campbell, 1988; Dunlosky \& Salthouse, 1996). Furthermore, there is evidence to suggest that there are age-related deficits in the computational aspects of learning (e.g., Kramer, Larish, \& Strayer, 1995; Strayer \& Kramer, 1994) and that older adults learn new associative relations less readily than do younger adults (e.g., J. E. Fisk \& Warr, 1998; Salthouse, 1994). However, relatively little is known about the extent to which different-aged adults come to rely on representation and retrieval of problem instances in lieu of computation in simple cognitive tasks in which items are repeated. In the present experiment we gave many repetitions of a small number of alphabet arithmetic problems to younger and older adults for the purpose of describing possible age differences in the development of a reliance on item retrieval in lieu of computation.

Logan's (1988) instance theory provided the rationale for examining the effects of repetitions of problem instances on cognitive skill acquisition. The basic idea of instance theory is that there is a shift in how problems are solved as a function of repetitions of problem instances. For many cognitive tasks, initial performance is assumed to be relatively slow because it depends on the execution of an algorithm for carrying out the task. As problem instances are repeated, instance theory predicts that there will be an increase in the probability that solutions will be retrieved from memory rather than computed. According to instance theory, there is a race between algorithmic processing and the retrieval of problem instances. The winner of the race determines the speed of performance on any given trial. To date, only a few studies have investigated instance-based learning in older adults (e.g., Jenkins \& Hoyer, 2000; Lincourt, Hoyer, \& Cerella, 1997).

A second aim of the present experiment was to examine possible age differences in the transfer of prior learning to the acquisition of a new set of problem instances. Some amount of improvement in skill acquisition can be the result of increased efficiency or speed of execution of the algorithm for solving problems in the task domain. However, most studies with young adults have reported that skilled performance is highly stimulus specific with little transfer to new problem sets (e.g., Singley \& Anderson, 1989; Speelman \& Kirsner, 1997). It seems that there might be even less transfer of learned skills across problem sets for older adults than for younger adults (e.g., Charness \& Campbell, 1988; A. D. Fisk, Cooper, Hertzog, \& AndersonGarlach, 1995; Rogers, 1992). In the present experiment, participants were given extensive 
practice with two sets of problem instances. It was assumed that the rate of improvement observed in Set 1 would reflect both algorithmic learning and the process of item retrieval and that acquisition performance in Set 2 would reflect primarily the process of item retrieval because algorithmic learning would already be at its maximum.

The third aim of the present experiment was to describe possible age differences in the effects of task requirements on acquisition. In studies using alphabet arithmetic tasks, participants are required either to produce answers to problems by making a vocal or manual response corresponding to a solution or to verify the solutions to equations as true or false (e.g., see Campbell, 1987; Campbell \& Tarling, 1996; Kramer et al., 1995; Zbrodoff \& Logan, 2000). Compared with conditions that require participants to produce responses, performance under verification conditions is composed of solution production plus comparison of the produced response with the given solution. Task differences in the processing steps involved in production and verification provide an opportunity to examine possible age differences in the effects of task requirements on measures of acquisition. In the present study, different groups of young adults and older adults were required to either produce or verify solutions to alphabet arithmetic problems. Because verification includes production as well as mental comparison of the generated and presented solutions, we expected that response times for older adults would be differentially longer for verification than for production because of age-related slowing associated with carrying out the additional processing step of verification. In addition, the use of different versions of the alphabet arithmetic task provided a basis for examining the generalizability of findings across versions.

Consistent with the analytic approach used in most investigations of the effects of aging and practice on cognitive skill acquisition, we compared the performance of younger and older adults by using measures of response time and accuracy of response. Unlike most previous studies, however, we also assessed rate of learning, improvement span (or the amount of practice required to reach asymptote), and asymptotic performance by using parameters representing these aspects of acquisition derived from fits of individual data to power functions. The power function is one of a class of nonlinear functions that depicts negatively accelerated improvement in performance as a function of practice. It has been demonstrated that acquisition data taken from a wide variety of task domains are well fit by a power function (e.g., Logan, 1988, 1992; Newell \& Rosenbloom, 1981; Rickard, 1997). [1] For the power function RT = a + $b \mathrm{~N}^{-c}$, where $\mathrm{RT}$ is response time and $\mathrm{N}$ is the number of practice trials, the a parameter represents the asymptotic performance, the $b$ parameter represents improvement span, and the $c$ parameter represents rate of change.

Parameter values derived from individual data can be averaged for the purposes of group comparisons. For illustration, Figure 1A shows two hypothetical power-function curves that differ in terms of the values of the $a, b$, and $c$ parameters. Curve 1 might resemble the performance of a group of older adults, and Curve 2 might resemble the performance of a group of younger adults. A difference in the a parameter indicates that the groups have different levels of maximal performance. An age effect in asymptote (e.g., $a_{\text {old }}>a_{\text {young }}$ ) may be attributed to a sensorymotor difference or to a diffuse or general slowing overhead (e.g., see Cerella, 1990). Obtained differences in the $b$ parameter are produced by differences in starting values and indicate the amount of improvement that can be brought about by practice. The starting value is calculated by adding the $b$ parameter and the a parameter. Obtained differences in the exponents of the power function, $c$, indicate differences in the learning rate (e.g., $c_{\text {old }}<c_{\text {young }}$ ). 

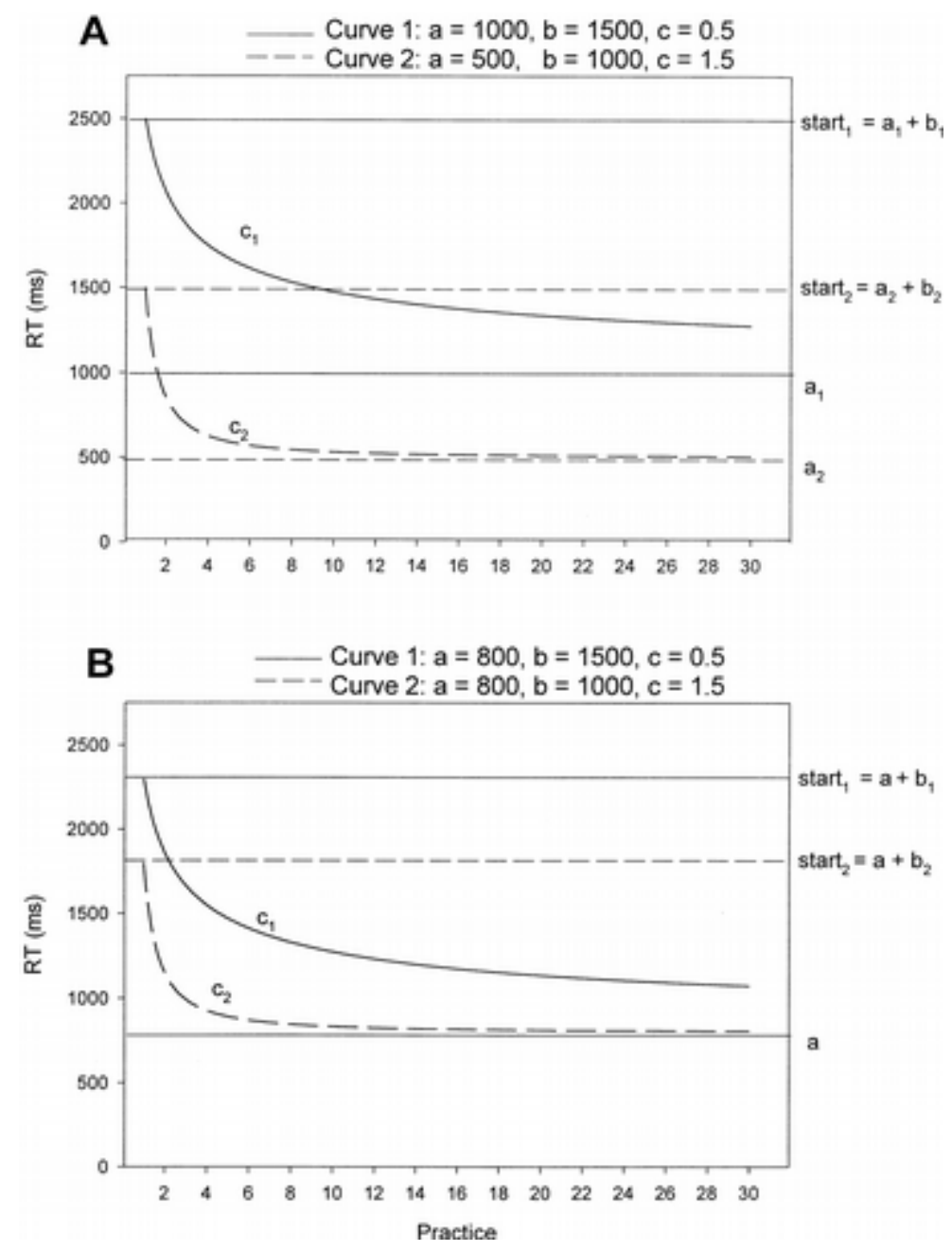

Figure 1. A: Examples of curves having different $a, b$, and c parameters. B: Examples of curves having a shared a parameter and distinct $b$ and $c$ parameters. $R T=$ response time; $a=$ asymptotic performance parameter; $b=$ improvement span parameter; $c=$ rate of change parameter

Possible age differences in learning can be described by using parameters derived from fits to individual data to specify a composite power-function model for grouped data. The general analytic method developed by Fisher and Glaser (1996) is applicable to any task for which an information-processing model is available in closed form: RT $=f(a, b, c, \vdots)$, where $a, b, c, \ldots$, are model parameters. For this multistep method, the model is fitted to data for younger and older participants ( $\left.\mathrm{RT}_{\text {young }} \mathrm{RT}_{\text {old }}\right)$ simultaneously. In the first round, the younger and older models are fully differentiated-

$$
\left\{\begin{array}{l}
\mathrm{RT}_{\text {young }}=f\left(a_{\text {young }}, b_{\text {young }}, c_{\text {young }}, \cdots\right) \\
\mathrm{RT}_{\text {old }}=f\left(a_{\text {old }}, b_{\text {old }}, c_{\text {old }}, \cdots\right)
\end{array}\right\},
$$


with each data set described by its own parameters. This round establishes the maximum amount of variance that is systematic with respect to the model. Parameters that do not represent statistically reliable differences can be collapsed across groups, allowing for a more highly constrained model with fewer parameters. In subsequent steps, shared parameters are introduced; for example,

$$
\left\{\begin{array}{l}
\mathrm{RT}_{\text {young }}=f\left(a_{\text {young }}, b_{\text {young }}, c, \cdots\right) \\
\mathrm{RT}_{\text {old }}=f\left(a_{\text {old }}, b_{\text {old }}, c, \cdots\right)
\end{array}\right\} .
$$

Figure 1B illustrates two curves with a shared a parameter and separate $b$ and $c$ parameters. Reducing the number of free parameters invariably reduces the amount of variance that can be accounted for in the model. An incremental $F$ test can be used to determine if the reduction in amount of variance accounted for is reliable given the reduced degrees of freedom. A nonsignificant $F$ test can be interpreted as suggesting that the groups do not differ on the shared parameters. [2] These analytic procedures are used to identify the sources of age-group differences in the acquisition functions and in the transfer of Set 1 learning.

\section{METHOD Participants}

The participants were 24 young adults between the ages of 18 and 21 years and 24 older adults between the ages of 64 and 75 years (young: $M=19.12$, $S D=0.74$; older: $M=70.54, S D=$ 3.31). Equal numbers of young and older adults were assigned to the verification condition and the production condition. Young adults were recruited from the human subjects pool in the Department of Psychology at Syracuse University and received partial course credit for their participation. Older adults were community-residing volunteers who were recruited from newspaper advertisements or from the registry of the Adult Cognition Laboratory in the Department of Psychology at Syracuse University. Older adults received a $\$ 30$ honorarium for their participation.

Participants reported their medication usage and rated their overall health status, their physical limitations in daily activities, and their comfort level with computer use on a 5-point scale. Individuals who reported that they were not taking any medications known to affect memory or attention and who rated their health, physical activity level, and comfort level with computers as good or excellent (ratings of 2 or 1, respectively) were eligible to participate in the study. Individuals were screened for near visual acuity and were excluded from participation if acuity was worse than $20 / 30$ (with normal correction).

Several brief tests of memory, perceptual speed, and numerical speed were administered for the purpose of describing the age samples. Mean scores for the Digit Span and Digit Symbol Substitution subtests of the Wechsler Adult Intelligence Scale- Revised (Wechsler, 1981), the Number subtest from the Primary Mental Abilities Test (Thurstone \& Thurstone, 1949), the Number Comparisons subtest from the Kit of Factor-Referenced Cognitive Tests (Ekstrom, French, Harman, \& Dermen, 1976), and for other characteristics of the samples are reported in Table 1. 
Table 1

Characteristics of the Participants

\begin{tabular}{|c|c|c|c|c|}
\hline \multirow[b]{2}{*}{ Measure } & \multicolumn{2}{|c|}{ Young } & \multicolumn{2}{|c|}{ Older } \\
\hline & $M$ & $S D$ & M & $S D$ \\
\hline Education (years)* & 12.88 & 0.54 & 14.42 & 2.28 \\
\hline Computer use & 3.92 & 0.93 & 3.46 & 1.35 \\
\hline Health & 2.04 & 1.12 & 2.20 & 0.78 \\
\hline Limitations & 1.33 & 0.70 & 1.79 & 0.24 \\
\hline Digit span & 15.71 & 3.71 & 15.58 & 3.37 \\
\hline Digit symbol** & 67.79 & 9.19 & 52.83 & 12.98 \\
\hline Vocabulary* & 30.21 & 3.35 & 35.42 & 3.19 \\
\hline Number comparison & 23.42 & 5.98 & 19.08 & 9.81 \\
\hline PMA number calculation* & 22.42 & 7.05 & 32.67 & 12.60 \\
\hline
\end{tabular}

Note. PMA $=$ Primary Mental Abilities test.

$* p<.05$.

Characteristics of the Participants

\section{Materials and Procedure}

Presentation of the stimulus displays and measurement and recording of response times and errors were controlled by a Visual Basic computer program. The participants' task was to either verify alphabet arithmetic equations (by pressing the $Z$ key on the keyboard to indicate "true" and the "l" key to indicate "false") or to produce the answer to alphabet arithmetic equations (by pressing the number key corresponding to the correct answer). Each verification problem was an alphabet arithmetic equation consisting of a single-digit addend (either a 1, 2, 3, 4, or 5), a plus sign, an uppercase letter (either an A, B, C, D, or E), an equal sign, and a single digit (e.g., $3+A=4)$. Half the equations were true, and half were false. False answers were one number higher than the true answer (if this rule produced a two-digit answer, a false answer that was one number below the true answer was used). Each production problem was an alphabet arithmetic question to be solved and consisted of a single-digit addend (either a 1, 2, 3, 4, or 5), a plus sign, an uppercase letter (either an A, B, C, D, or E), an equal sign, and a question mark (e.g., $3+A=$ ?). Participants were instructed to produce or verify answers to the problems by first converting the letter to a digit (e.g., $A=1)$ and then adding the digits. Problems were presented horizontally in the center of the computer screen at eye level and subtended approximately $4^{\circ}$ of visual angle at a viewing distance of $90 \mathrm{~cm}$. The list of problems used in this experiment is provided in Appendix $A$.

Participants were tested individually in a quiet laboratory room. Instructions were presented on the computer screen and were read orally by the experimenter. In the instructions, examples demonstrated solving alphabet arithmetic equations by converting the letter to a number and then adding. Instructions emphasized both speed and accuracy of responding. Participants assigned to the verification condition were given 40 arithmetic practice trials of the form $3+2=$ 5 at the beginning of each session. Participants assigned to the production condition were given 40 arithmetic practice trials of the form $3+2=$ ? at the beginning of each session. Because of the response requirements in the production condition, participants assigned to this condition were also given number pad practice consisting of 75 trials requiring a key-press response to single digits at the beginning of each session. 
Alphabet arithmetic trials were administered immediately after completion of the practice trials. One set of stimuli was administered in each session. The order of presentation of the stimulus sets was counterbalanced within age groups. In each session there was a total of 600 experimental trials grouped into 20 blocks of 30 trials. Each block contained five repetitions of the same six problems (for verification problems, three true and three false problems were given). Procedures were identical for both sessions except that a different set of problems was administered in each session.

A trial consisted of the presentation of a fixation cross in the center of the screen for $500 \mathrm{~ms}$, followed by the presentation of an alphabet arithmetic problem that remained on the screen until a response was made. Error responses were followed by an "error" message in the center of the screen. Both correct and error responses were followed by a 1,000-ms intertrial interval. Participants were given the opportunity to take a rest break after each block of trials.

\section{RESULTS}

Response times longer than 7,000 ms or shorter than $200 \mathrm{~ms}$ were considered outliers and were removed, resulting in the removal of less than .02\% of responses. Mean correct response times were calculated for each age group and problem set by blocks; these data are presented in Figure 2. The blocks variable was reorganized from 20 blocks of 30 trials to 10 blocks of 60 trials for statistical analysis and for clarity of presentation.

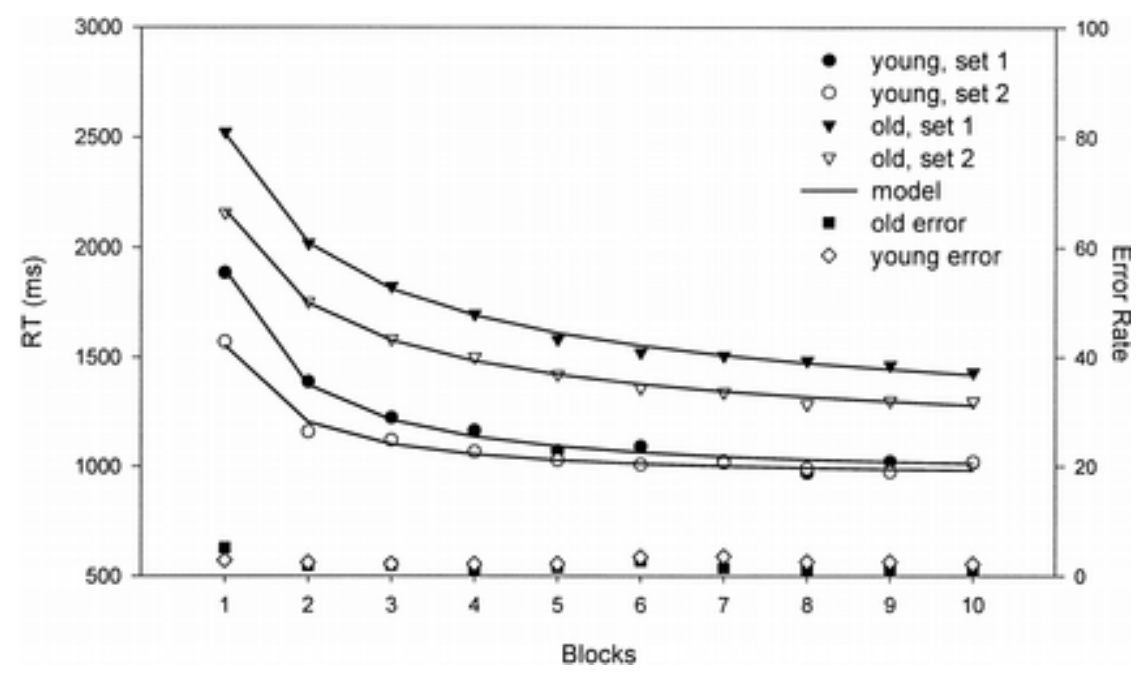

Figure 2. Fits for a nine-parameter composite model by age and problem set, and mean error rates by age. $R T=$ response time

Response times were analyzed in three ways. First, an analysis of variance (ANOVA) was used to describe possible differences associated with age (young, older), problem set (first, second), task (verification, production), and training (Blocks 1-10). Second, response times for each 
participant were fit to power functions and an ANOVA was used to describe possible differences associated with age, problem set, and task in the $a, b$, and $c$ parameters of the power functions. Third, power functions were fit to the group means and composite models were constructed guided by the parameters for which there were statistically reliable group differences. Effect sizes for the analysis of response times and for the analysis of the parameter values are reported for all single-degree-of-freedom $F$ tests by using a form of Pearson's $r$, as discussed by Rosenthal and Rosnow (1985). The estimated power to detect an effect at the .05 level was .75 or higher for the analysis of response times, .65 or higher for the analysis of the parameter values, and .75 or higher for the analysis of the accuracy data (see Cohen, 1988).

\section{ANOVA for Response Times}

An ANOVA for mean response times revealed expected main effects of age (young: $M=1,140$ ms, $S D=466$; older: $M=1,602 \mathrm{~ms}, S D=647), F(1,46)=12.08, p=.0011, M S E=4,241,237, r$ $=.46$; problem set (Set 1: $M=1,445 \mathrm{~ms}, S D=657$; Set 2: $M=1,297 \mathrm{~ms}, S D=548), F(1,46)=$ 24.53, $p<.0001, M S E=213,034, r=.59$; and block (Block 1: $M=2,033 \mathrm{~ms}$, $S D=747$; Block 10: $M=1,192 \mathrm{~ms}, S D=493), F(9,414)=137.32, p<.0001, M S E=49,250$. These main effects were qualified by an Age $\times$ Block interaction, $F(9,414)=4.41, p<.0001, M S E=49,250$, and by a Problem Set $\times$ Block interaction, $F(9,414)=8.78, p<.0001, M S E=20,073$. No main effects or interactions with the task variable were observed. Comparisons of the group means suggested that improvement from Set 1 to Set 2 was greater for older adults than for younger adults, but the interaction of age and problem set did not reach statistical significance, $F(1,46)$ $=3.76, p=.059$, MSE $=213,034, r=.27$. The group means by age and problem set were as follows (standard deviations are shown in parentheses): young, Set 1 = 1,185 ms (489 ms); young, Set 2 = 1,095 ms (437 ms); older, Set $1=1,705 \mathrm{~ms}$ (699 ms); and older, Set $2=1,499$ $\mathrm{ms}(573 \mathrm{~ms})$. The significant Age $\times$ Block interaction indicated that improvement was greater for older adults than for young adults. Means for the Age $\times$ Block comparisons were as follows (standard deviations are shown in parentheses): young, Block $1=1,727 \mathrm{~ms}$ (492 ms); young, Block 10 = 1,019 ms (359 ms); older, Block 1 = 2,339 ms (829 ms); and older, Block $10=1,365$ ms (527 ms). The Problem Set $\times$ Block interaction indicated that improvement over blocks was greater in Problem Set 1 than in Problem Set 2. The means for this interaction were as follows (standard deviations are shown in parentheses): set 1, Block $1=2,204 \mathrm{~ms}$ (747 ms); Set 1, Block $10=1,224 \mathrm{~ms}$ (493 ms); Set 2, Block $1=1,863 \mathrm{~ms}$ (671 ms); and Set 2, Block $10=1,159$ $\mathrm{ms}$ (442 ms). Thus, the results of the ANOVA point to the conclusion that improvement was greatest for older adults and for Set 1 learning. The improbable conclusion that improvement was greater for older adults than for younger adults prompted an examination of the forms of learning functions.

\section{ANOVA for the Parameters of the Power Functions}

To examine age, set, and task differences in the forms of the learning functions, the block-byblock data for each problem set and task for each participant were fit to three-parameter power functions. Mean goodness-of-fit values (percent variance explained) for the young adults and the older adults were $82.7 \%$ and $86.7 \%$ respectively. An ANOVA formed on these $R^{2}$ values indicated that there was no group difference in the degree of fit of the power functions. The worst fit of the 48 data sets was $33 \%$, residuals were examined and did not appear to be 
systematic, and none of the $48 \times 3(144)$ parameter values was wildly implausible. Consistent with the results of the ANOVA for response times, the ANOVA for the parameters of the power functions revealed no effects of task (verification vs. production), so we collapsed across task for the analyses reported below.

Means for the $a, b$, and $c$ parameters for young adults and older adults are plotted by problem set in the three panels of Figure 3. Age and set differences in asymptotic performance and improvement span are indicated by the $a$ and $b$ parameters in Panels $A$ and $B$, respectively, and differences in the rate of learning are indicated by the $c$ parameters shown in Panel $C$. The effects of age and problem set on the $a, b$, and $c$ parameters were examined in separate ANOVAs. The analysis of asymptotes indicated a main effect of age, $F(1,44)=6.29, p=.0159$, $M S E=306,674, r=.35$. As expected, the response time representing asymptotic performance for the young adults was shorter than the response time representing asymptotic performance for older adults. This finding indicates that the overall slower performance of older adults was not eliminated with extensive practice. The analysis of asymptotes revealed no main effects of problem set or interactions of age with set. 

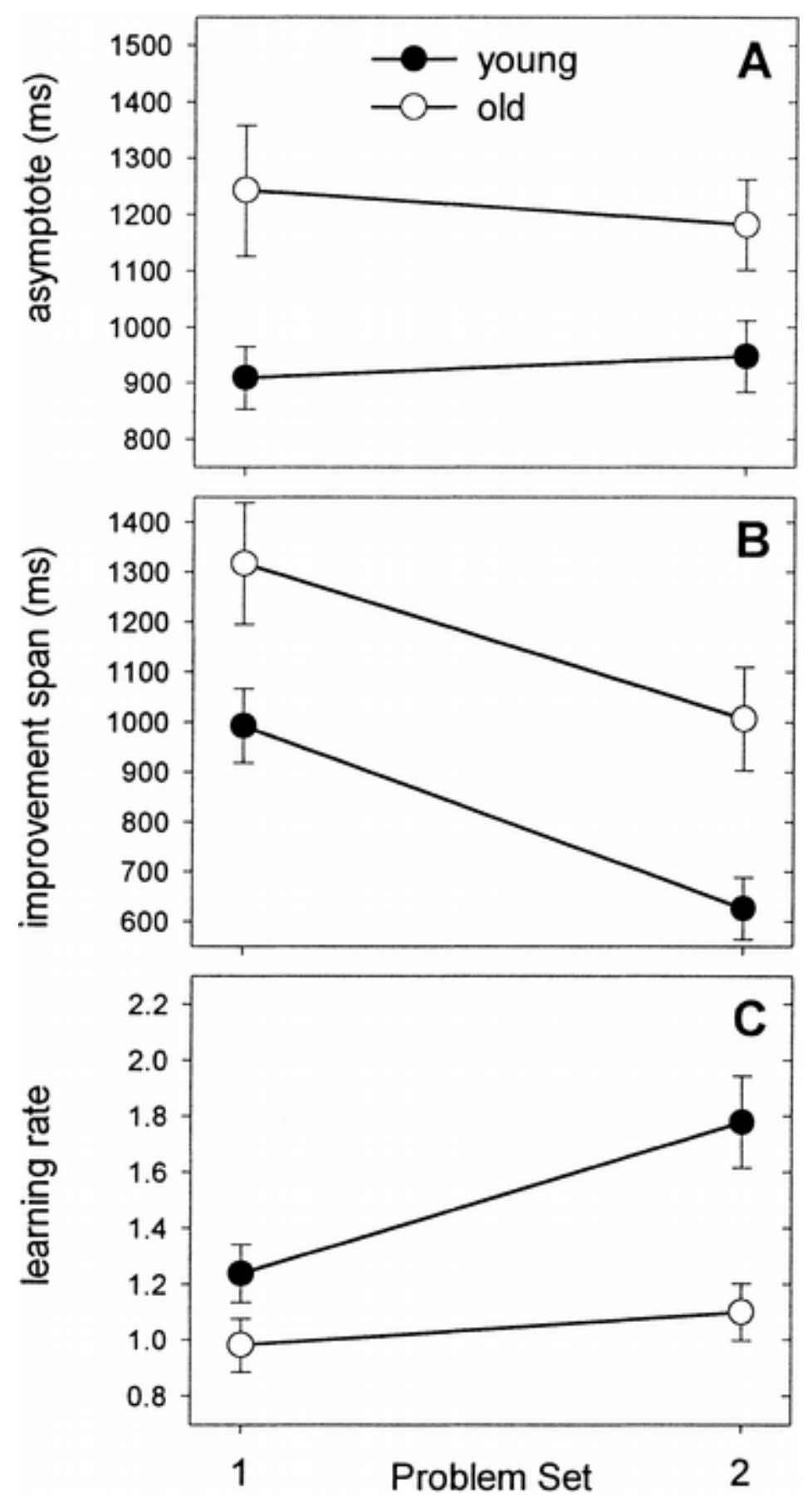

Figure 3. Means (and standard errors) for the parameters of the power-function fits by age and problem set. A: Asymptotes. B: Improvement spans. C: Learning rates

The analysis of improvement spans indicated main effects of age, $F(1,44)=9.11, p=.0042$, $M S E=327,625, r=.41$, and problem set, $F(1,44)=32.36, p<.0001, M S E=84,861, r=.65$. The amount of learning required to reach asymptote was smaller for young adults than for older adults, and was smaller for Problem Set 2 than for Problem Set 1. The smaller improvement 
spans in Set 2 are due to differences in the initial response times for Sets 1 and 2. That initial response times were shorter for Set 2 than for Set 1 can be attributed to the general benefits of computational practice. In both problem sets, initial response times were longer for older adults than for younger adults. The Age $\times$ Problem Set interaction was not significant for the improvement span measure.

Analysis of the learning rates (the $c$ parameters) indicated that there were main effects of age, $F(1,44)=10.98, p=.0018, M S E=0.4779, r=.45$, and problem set, $F(1,44)=11.12, p=$ $.0017, M S E=0.2345, r=.45$. The rates of learning were faster for younger adults than for older adults and were faster in Problem Set 2 than in Problem Set 1. The improvements in learning rates observed for Set 2 indicate the benefit of computational practice on subsequent acquisition. The Age $\times$ Problem Set interaction was reliable, $F(1,44)=4.52, p=.0392, M S E=$ $0.2345, r=.31$. Improvement in learning rates in Set 2 were greater for younger adults, indicating a greater benefit of prior learning for young adults than for older adults.

Correlations for the parameter values are reported in Table 2. None of the correlations between different parameters reached significance, suggesting that each parameter indexed a distinct aspect of the effects of practice on performance. The correlations between the same parameters in different problem sets were reliable for the $a$ and $b$ parameters for both age groups, suggesting that these measures are consistent across problem sets. In contrast, the lack of a correlation between problem sets for the $c$ parameter corroborates the results of the ANOVA and suggests a between-sets difference in the type or efficiency of processing.

Table 2

Pearson Product-Moment Correlations for Parameter Values by Set for Young and Older Adults

\begin{tabular}{|c|c|c|c|c|c|}
\hline Parameter and set & 2 & 3 & 4 & 5 & 6 \\
\hline \multicolumn{6}{|c|}{ Young } \\
\hline $\begin{array}{l}\text { 1. } a_{\mathrm{Set} 1} \\
2 . a_{\mathrm{Set} 2} \\
\text { 3. } b_{\mathrm{Set} 1} \\
\text { 4. } b_{\mathrm{Set} 2} \\
\text { 5. } c_{\mathrm{Set} 1} \\
6 \cdot c_{\mathrm{Set} 2}\end{array}$ & $\frac{.79^{*}}{-}$ & $\begin{array}{l}.16 \\
.20 \\
-\end{array}$ & $\begin{array}{c}.45 \\
.37 \\
.71^{*} \\
-\end{array}$ & $\begin{array}{l}-.40 \\
-.44 \\
-.21 \\
-.40 \\
-\end{array}$ & $\begin{array}{r}-.20 \\
-.09 \\
-.37 \\
-.39 \\
.32 \\
- \\
\end{array}$ \\
\hline \multicolumn{6}{|c|}{ Older } \\
\hline $\begin{array}{l}\text { 1. } a_{\mathrm{Set} 1} \\
\text { 2. } a_{\mathrm{Set} 2} \\
\text { 3. } b_{\mathrm{Set} 1} \\
\text { 4. } b_{\mathrm{Set} 2} \\
\text { 5. } c_{\mathrm{Set} 1} \\
\text { 6. } c_{\mathrm{Set} 2}\end{array}$ & $\frac{.86^{\circ}}{-}$ & $\begin{array}{l}.19 \\
.41 \\
-\end{array}$ & $\begin{array}{r}.50 \\
.36 \\
.58^{\mathbf{}} \\
-\end{array}$ & $\begin{array}{r}.32 \\
.08 \\
-.34 \\
-.01 \\
-\end{array}$ & $\begin{array}{r}-.15 \\
-.13 \\
-.17 \\
-.14 \\
.33 \\
-\end{array}$ \\
\hline
\end{tabular}

Note. Correlations for the same parameters between sets are in bold. $a=$ asymptotic performance parameter; $b=$ improvement span parameter; $c=$ rate of change parameter. $* p<.01$.

Pearson Product-Moment Correlations for Parameter Values by Set for Young and Older Adults 


\section{Composite Models for Group Response Times}

A composite data set was assembled consisting of the group means for each age group and condition broken down by block and collapsed across problem type. The resulting four curves are shown in Figure 2. Power functions were fit to the four curves simultaneously. The resulting 12-parameter model ( 4 curves $\times 3$ parameters per curve) accounted for $99.74 \%$ of the variance in the combined data set. The parameter values are shown in Table 3. The results of an ANOVA suggested that the composite data set may be satisfactorily described by fewer than 12 distinct parameters. For example, the asymptote parameters for individuals differed with age but not with problem set, and the Age $\times$ Set interaction was not reliable. This finding suggests that a distinct asymptote parameter for each of the curves (young set 1 $_{1}$, young set 2 $_{2}$, old set 1 $_{1}$, old set 2 $_{\text {2 }}$ may be unnecessary at the group level. A reduced model was therefore constructed using only two distinct a parameters ( $a_{\text {young }}$ and $\left.a_{\text {age offset }}\right)$. Note that the offset parameter is used to represent the amount of difference associated with a main effect. The improvement-span parameters for individuals differed both with age and with problem set, but without an Age $\times$ Set interaction, suggesting that the four $b$ parameters of the group curves could be reduced to three $\left(b_{\text {young }}, b_{\text {age }}\right.$ offset, and $\left.b_{\text {problem set offset }}\right)$. Because age, set, and the Age $\times$ Set interactions were all significant for the rate parameters for individuals, it seemed likely that four $c$ parameters would be necessary to describe the group curves ( $C_{\text {young, Set 1 }}, C_{\text {young, Set 2 }}, C_{\text {old, Set } 1}, C_{\text {old, Set } 2}$ ). The reduced model contained nine parameters (see Appendix B for equations). Fitted to the data, the nineparameter model accounted for $99.68 \%$ ofthe variance (and is illustrated in Figure 2). The difference in the amount of variance accounted for by the reduced model and the full model was $.06 \%$. An incremental $F$-test indicated that the additional variance explained by the 12parameter model was not significant when weighted against the additional parameters, $F(3,27)$ $=9.372, p=.005$. Thus, the reduced model indeed provided an adequate description of the group data. 
Table 3

Values for the 12-Parameter and 9-Parameter Composite Models $\left(R T=a+b \mathrm{~N}^{-}\right.$)

\begin{tabular}{lc}
\hline \multicolumn{1}{c}{ Model } & Value \\
\hline \multicolumn{1}{c}{ 12-parameter model } \\
$a_{\text {young, Set 1 }}$ \\
$a_{\text {young, Set 2 }}$ & 898 \\
$a_{\text {old, Set 1 }}$ & 979 \\
$a_{\text {old, Set 2 }}$ & 1,101 \\
$b_{\text {young, Set 1 }}$ & 1,006 \\
$b_{\text {young, Set 2 }}$ & 987 \\
$b_{\text {old, Set 1 }}$ & 587 \\
$b_{\text {old, Set 2 }}$ & 1,425 \\
$c_{\text {young, Set 1 }}$ & 1,153 \\
$c_{\text {young, Set 2 }}$ & 1.009 \\
$c_{\text {old, Set 1 }}$ & 1.514 \\
$c_{\text {old, Set 2 }}$ & 0.633 \\
& 0.642 \\
\hline \multicolumn{1}{c}{9 -parameter model } \\
$a_{\text {young }}$ & 947 \\
$a_{\text {age offset }}$ & 98 \\
$b_{\text {young }}$ & 952 \\
$b_{\text {age offset }}$ & 517 \\
$b_{\text {problem set ofset }}$ & -346 \\
$c_{\text {young, Set 1 }}$ & 1.165 \\
$c_{\text {young, Set 2 }}$ & 1.246 \\
$c_{\text {old, Set 1 }}$ & 0.594 \\
$c_{\text {old, Set 2 }}$ & 0.682 \\
\hline
\end{tabular}

Note. $\mathrm{RT}=$ response time; $\mathrm{N}=$ number of practice trials; $\quad a=$ asymptotic performance parameter; $b=$ improvement span parameter; $c=$ rate of change parameter.

Values for the 12-Parameter and 9-Parameter Composite Models $(R T=a+b N-c)$

\section{Accuracy of Responses}

The overall level of accuracy was high (97.7\%) and never dropped below 94\%. An ANOVA revealed a main effect of block, $F(9,396)=6.78, p<.0001, M S E=0.00125$, and an Age $\times$ Block interaction, $F(9,396)=3.12, p=.0012, M S E=0.00125$. Increases in accuracy across blocks were greater for older adults than for younger adults (see Figure 2). No other main effects or interactions were found. The possibility of a speed-accuracy trade-off by age can be dismissed by inspection of the accuracies and response times shown in Figure 2. Note that a substantial amount of the improvement in accuracy for older adults occurred in the first block of training.

\section{Comparison of Verification Data and Production Data}

Results of the present study also bear on the effects of age on the processing components of verification and production. It has been reported that participants perform the verification task by first producing an answer and then comparing it with the presented answer, saying "true" if it 
matches and "false" if it does not. Because verification includes production as well as mental comparison of the generated and presented solutions, it was expected that response times would be longer for verification than for production and that the verification response times for older adults would be especially longer because of age-related slowing associated with carrying out the additional step of verification. Contrary to expectation, there was no evidence to suggest differences between the processing demands of production and verification. Rather, the verification data and production data can be taken as replications that serve to increase confidence in the findings regarding age and problem-set effects. Unfortunately, a presumably minor procedural difference in the amount of preexperimental practice given to participants in the verification and production groups confuses the interpretation of this noneffect. That is, all participants were given the same amount of preexperimental practice with production or verification of alphabet arithmetic solutions, but participants in the production condition were given a small amount of additional practice in using the number pad. Although this procedural difference possibly gave a very slight initial advantage to participants in the production condition, it probably contributed little or not at all to the experimental measures.

\section{Discussion}

Current theories of cognitive skill acquisition tie improvements with practice to computational speedup and to the retrieval of accumulated problem instances. Benefits associated with computational speedup as well as with item retrieval in lieu of computation can lead to faster overall response times. Comparisons of the parameters of the power functions for Set 1 and Set 2 suggest the degree of benefit associated with the algorithmic learning and with the retrieval of problem instances, and provide a basis for describing the extent to which there are age differences in item retrieval and computational speedup. First, the difference in initial response times for Set $1(M=2,233 \mathrm{~ms}, S D=791)$ and Set $2(M=1,880 \mathrm{~ms}, S D=703)$ indicates that there was transfer of experience in using the alphabet arithmetic algorithm. Second, the difference between asymptotes for Set $1(M=1,076 \mathrm{~ms}, S D=473)$ and initial response times for Set $2(M=1,880 \mathrm{~ms}, S D=703)$ indicates the degree of benefit attributable to the use of retrieval of problem instances. These differences were statistically reliable: algorithmic learning, $F(1,46)=50.97, p<.0001, M S E=54,635$; retrieval of instances, $F(1,46)=137.01, p<.0001$, $M S E=71,441$. Because the contribution of algorithmic learning was at maximum in Set 2 , the learning rates observed in Set 2 reflect primarily or entirely the process of retrieving problem instances. The lack of a correlation between sets for the rate parameters for both age groups is consistent with this interpretation. The relatively large improvement in learning rate for young adults in Set 2 indicates a more pronounced benefit of computational practice on subsequent learning for young adults than for older adults.

Clearly, the analyses of fits of the parameters of the power functions provided a more comprehensive and detailed description of the effects of aging on cognitive skill acquisition than did the analysis of response times per se. The findings we report regarding age differences in the learning processes underlying cognitive skill acquisition were invisible in the ANOVA for response times. Specifically, age differences in the amounts and rates of improvement were not 
found in the results of the ANOVA because differences in starting values and asymptotes were not simultaneously considered. Moreover, the pattern of interaction effects that was observed in the ANOVA for response times had the potentially misleading implication that learning rates were faster for older adults than for younger adults. Thus, studies that do not provide assessments of the parameters of nonlinear functions by age or that do not take into account baseline differences for different age groups can sometimes produce unclear or misleading findings. The results reported here add to a scant research literature and provide a detailed description of the sources of the effects of aging on cognitive skill acquisition.

Analysis of composite models proved to be an especially useful tool for describing the effects of aging on the processes of learning. Specifically, the constrained model represented the main findings that rates of learning were slower for older individuals than for younger individuals and that prolonged practice would not bring older adults to the same level of asymptote achieved by young adults. Further, the composite model represented the findings that asymptotic levels for younger and older adults in Set 2 were unaffected by Set 1 practice and that the age differences in skill acquisition observed in Set 2 were associated with learning rates for item retrieval. Age differences in initial response times in Set 2 suggested that the transfer of algorithmic learning and the benefit of Set 1 practice were greater for young adults than for older adults. The constrained model that collapsed across nonsignificant parameters in the full model, as well as the full 12-parameter model, accounted for more than $99 \%$ of the variance.

\section{FOOTNOTES}

1. Despite the demonstrated ubiquity of the power function, there is some research to suggest that fits to data using exponential functions and hyperbolic functions may do equally well or possibly better than power functions (see Heathcote, Brown, \& Mewhort, 2000; Mazur \& Hastie, 1978; Myung, Kim, \& Pitt, 2000; Van Zandt, 2000).

2. The inferential statistics must be interpreted cautiously in cases in which the model is not linear with respect to the parameters in question.

\section{REFERENCES}

Campbell, J. I. D. (1987). Production, verification, and priming of multiplication facts. Memory \& Cognition, 15, 349-364.

Campbell, J. I. D., \& Tarling, D. P. M. (1996). Retrieval processes in arithmetic production and verification. Memory \& Cognition, 24, 156-172.

Cerella, J. (1990). Aging and information-processing rate. In J. E.Birren \& K. W.Schaie (Eds.), Handbook of the psychology of aging (3rd ed., (pp. 201-221). New York: Academic Press.

Charness, N., \& Campbell, J. I. D. (1988). Acquiring skill at mental calculation in adulthood: A task decomposition. Journal of Experimental Psychology: General, 117, 115-129. 
Cohen, J. (1988). Statistical power analysis for the behavioral sciences (2nd ed.). Hillsdale, NJ: Erlbaum.

Dunlosky, J., \& Salthouse, T. A. (1996). A decomposition of age-related differences in multitrial free recall. Aging, Neuropsychology, and Cognition, 3, 2-14.

Ekstrom, R. B., French, J. W., Harman, H., \& Dermen, D. (1976). Manual and kit of factorreferenced cognitive tests. Princeton, NJ: Educational Testing Service.

Fisher, D. L., \& Glaser, R. A. (1996). Molar and latent models of cognitive slowing: Implications for aging, dementia, depression, development, and intelligence. Psychonomic Bulletin and Review, 3, 458-480.

Fisk, A. D., Cooper, B. P., Hertzog, C., \& Anderson-Garlach, M. (1995). Age-related retention of skilled memory search: Examination of associative learning, interference, and task-specific skills. Journal of Gerontology: Psychological Sciences, 50B, P150-P161.

Fisk, J. E., \& Warr, P. P. (1998). Associative learning and short-term forgetting as a function of age, perceptual speed, and central executive functioning. Journal of Gerontology: Psychological Sciences, 53B, P112-P121.

Heathcote, A., Brown, S., \& Mewhort, D. J. K. (2000). The power law repealed: The case for an exponential law of practice. Psychonomic Bulletin and Review, 7, 185-207.

Jenkins, L., \& Hoyer, W. J. (2000). Instance-based automaticity and aging: Acquisition, reacquisition, and long-term retention. Psychology and Aging, 15, 551-565.

Kramer, A. F., Larish, J. F., \& Strayer, D. L. (1995). Training for attentional control in dual task settings: A comparison of young and old adults. Journal of Experimental Psychology: Applied, 1, 50-76.

Lincourt, A. E., Hoyer, W. J., \& Cerella, J. (1997, November). Effects of aging on the development of instance-based automaticity.Paper presented at the meetings of the Psychonomics Society, Philadelphia.

Logan, G. D. (1988). Toward an instance theory of automatization. Psychological Review, 95, 492-528.

Logan, G. D. (1992). Shapes of reaction-time distributions and shapes of learning curves: A test of instance theory of automatization. Journal of Experimental Psychology: Learning, Memory, and Cognition, 18, 883-914.

Mazur, J., \& Hastie, R. (1978). Learning as accumulation: A reexamination of the learning curve. Psychological Bulletin, 85, 1256-1274.

Myung, I. J., Kim, C., \& Pitt, M. A. (2000). Toward an explanation of the power law artifact: Insights from a response surface analysis. Memory \& Cognition, 28, 832-840. 
Newell, A., \& Rosenbloom, P. S. (1981). Mechanisms of skill acquisition and the law of practice. In J. R.Anderson (Ed.), Cognitive skills and their acquisition (pp. 1-56). Hillsdale, NJ: Erlbaum.

Rickard, T. C. (1997). Bending the power law: A CMPL theory of strategy shifts and the automatization of cognitive skills. Journal of Experimental Psychology: General, 126, 288-310.

Rogers, W. A. (1992). Age differences in visual search: Target and distractor learning. Psychology and Aging, 7, 526-535.

Rosenthal, R. L., \& Rosnow, D. B. (1985). Contrast analysis: Focused comparisons in the analysis of variance. Cambridge, England: Cambridge University Press.

Salthouse, T. A. (1994). Aging associations: Influence of speed on adult age differences in associative learning. Journal of Experimental Psychology: Learning, Memory, and Cognition, 20, 1486-1503.

Singley, M. K., \& Anderson, J. R. (1989). The transfer of cognitive skill. Cambridge, MA: Harvard University Press.

Speelman, C. P., \& Kirsner, K. (1997). The specificity of skill acquisition and transfer. Australian Journal of Psychology, 49, 91-100.

Strayer, D. L., \& Kramer, A. F. (1994). Aging and skill acquisition: Learning-performance distinctions. Psychology and Aging, 9, 589-605.

Thurstone, L. L., \& Thurstone, T. G. (1949). Examiner manual for the SRA Primary Mental Abilities Test. (Form 10-14). Chicago, IL: Science Research Associates.

Van Zandt, T. (2000). How to fit a response time distribution. Psychonomic Bulletin and Review, 7, 424-465.

Wechsler, D. (1981). Wechsler Adult Intelligence Scale—Revised. New York: Psychological Corporation.

Zbrodoff, N. J., \& Logan, G. D. (2000). When it hurts to be misled: A Stroop-like effect in a simple addition production task. Memory \& Cognition, 28, 1-7. 


\section{Appendix A}

Problem Sets Used in the Present Study

Set 1

True

False

Set 2

True

False

Set 1

Set 2

Verification

$$
\begin{aligned}
& 1+A=2 \\
& 1+A=3 \\
& 2+C=5 \\
& 2+C=6
\end{aligned}
$$

$1+\mathrm{A}=$ ?

$2+\mathrm{D}=$ ?

$2+\mathrm{C}=$ ?

$1+\mathrm{B}=$ ?

$$
\begin{aligned}
& 3+D=7 \\
& 3+D=8 \\
& 3+A=4 \\
& 3+A=5
\end{aligned}
$$

Production

$$
\begin{aligned}
& 3+B=? \\
& 3+E=? \\
& 3+D=? \\
& 5+A=?
\end{aligned}
$$

$5+\mathrm{C}=8$

$5+\mathrm{C}=9$

$4+\mathrm{E}=9$

$4+E=8$

$5+\mathrm{C}=$ ?

$4+C=$ ?

$4+\mathrm{E}=$ ?

$3+\mathrm{C}=$ ?

\section{Appendix B}

\section{Composite Power-Function Equations}

Twelve-Parameter Composite Power Function Equations

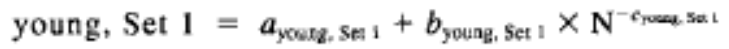

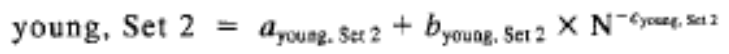

$$
\begin{aligned}
& \text { old, Set } 1=a_{\text {old, Sea } 1}+b_{\text {odd. Sea } 1} \times \mathrm{N}^{- \text {ead, Set } 1} \\
& \text { old, Set } 2=a_{\text {old, Set } 2}+b_{\text {old, Set } 2} \times \mathrm{N}^{-c_{\text {eth }} \text { Sed : }}
\end{aligned}
$$

\section{Nine-Parameter Composite Power-Function Equations}

$$
\begin{aligned}
& \text { young, Set } 1=a_{\text {young }}+b_{\text {young }} \times \mathrm{N}^{- \text {Gyores Set } 1} \\
& \text { young, Set } 2=a_{\text {young }}+\left(b_{\text {young }}+b_{\text {Set } 2}\right) \times N^{-c_{\text {your. Set } 2}} \\
& \text { old, Set } 1=\left(a_{\text {youag }}+a_{2 \text { ge offset }}\right)+\left(b_{\text {young }}+b_{\text {age offiset }}\right) \times \mathrm{N}^{-c_{\text {odd Se1 } 1}} \\
& \text { old, Set } 2=\left(a_{\text {yourg }}+a_{\text {age offset }}\right)+\left(b_{\text {ycuag }}+b_{\text {age offigt }}+b_{\text {Set } 2}\right) \times N^{-c_{\text {ald }} \text {. Se 2 }}
\end{aligned}
$$

